\title{
A small molecule improves Cas9 specificity
}

Making Cas9 nuclease activity dependent on the self-cleavage of a ligand-responsive intein decreases Cas9's off-target activity.

Specificity is a recurring theme in genome editing - the question is how to ensure high activity at the target site while not introducing modifications elsewhere in the genome. The strategies taken thus far for CRISPR (clustered, regularly interspaced, short palindromic repeats) editing optimize the selectivity of the guide RNA or use dimeric forms of Cas 9 that require two simultaneous binding events for a double-strand cut. David Liu from Harvard University has explored a strategy that limits the time window during which the Cas9 nuclease can cleave DNA.

Liu points out that genome editing is not a steady-state process. There are only two desired substrate molecules in each diploid cell; therefore, "once a genome-editing agent has catalyzed the two turnovers, the only things it can do are undesirable," says Liu. "If you limit exposure of a cell to an active genome-editing agent to a time window just long enough to achieve desired levels of on-target modification, you should increase the overall specificity of genome modification."

To exert temporal control over Cas9, Liu's graduate student Kevin Davis made use of an intein, developed in the Liu lab over a decade ago, that self-cleaves in response to hydroxytamoxifen (HT). Davis fused the intein to the Cas9 alpha-helical domain and showed that this fusion protein does not cleave DNA. Addition of HT triggers the splicing of the intein and leaves an activated Cas9. The team targeted endogenous loci in human cells and assessed the degree of nonhomologous end joining, a process used by the cell to repair cut DNA. They observed an increase in specificity of up to two orders of magnitude when Cas9 activity

\section{GENE EXPRESSION}

\section{HIGHLY MULTIPLEXED TRANSCRIPTOME IMAGING}

\section{A single-molecule imaging approach allows for error-robust transcriptomic} analyses in single cells.

Studying biology at the single-cell level can provide important insights into the role of differential gene expression in cellular structure and function. One way to study gene expression in single cells is to look at a cell's transcriptome, which is typically carried out using high-throughput sequencing. However, single-cell RNA sequencing methods can be error-prone and do not record the spatial organization of RNAs within a cell or tissue.

Xiaowei Zhuang and her team of researchers at Harvard University and the Howard Hughes Medical Institute wanted to develop a method for imaging the transcriptome of individual cells in situ. To do so, they developed multiplexed error-robust fluorescence in situ hybridization (MERFISH), a method that combines single-molecule fluorescence in situ hybridization (smFISH) with sequential imaging, combinatorial labeling and error-robust encoding to report the localization of hundreds to thousands of individual RNAs within their cellular and tissue context.

smFISH is a sensitive and accurate method for imaging RNAs that uses fluorescently labeled antisense probes against a target RNA. Attempts to multiplex smFISH have used combinations of probe colors. However, these approaches are limited by the colors available for imaging. Intrigued by this problem, Zhuang "came up with this idea that we could actually use the time domain to solve the problem." Instead of using multiple colors, the researchers label and image different subsets of RNAs over multiple rounds, treating each round as a pseudocolor. "This way we can simply image many rounds and use that to effectively increase the color space. If you use this pseudocolor idea and use combinations of the pseudocolors, then all of a sudden it becomes feasible to image and distinguish thousands, or even tens of thousands, of RNA species." 
was restricted to 12 hours. The researchers are now looking to modify genes associated with human diseases using this strategy.

It is still being debated exactly how specific CRISPR reagents are and how much specificity is required for a given research or therapeutic application. Although several studies have reported minimal offtarget cuts in cells and also in mice (see Correspondence on p479 in this issue), the recently published study presenting an edited human embryo highlighted-among the important ethical issues surrounding this work - the need to better understand the technology, as off-target genome modifications were extensive in the zygotes.

Liu emphasizes the need for "a test that relates, preferably in a quantitative way, the relationship between off-target genome modification and organismal consequences," akin to an Ames test that determines the mutagenic potential of chemical compounds. "It would help enormously," says Liu, "to know that if the ratio of on- to offtarget modification falls below a certain level for a certain application, one should do secondary tests to look for negative biological consequences."

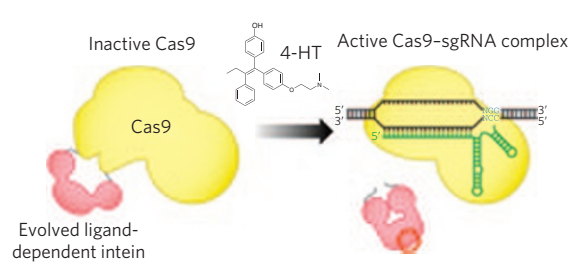

Addition of a ligand triggers intein self-cleavage and activates Cas9. Figure adapted from Davis et al., Nature Publishing Group.

But Liu sees reasons to be upbeat. Once the off-target mutations introduced by genome-editing tools are comparable to, or below, the natural rate of spontaneous mutation in healthy human cells, they are unlikely to increase toxicity to the cell or organism. How much improvement is needed to achieve this goal is at present unclear, but Liu is optimistic that a combination of approaches, such as engineered Cas9 variants and direct delivery of Cas9guide RNA complexes, will be successful.

\section{Nicole Rusk}

\section{RESEARCH PAPERS}

Davis, K.M. et al. Small molecule-triggered Cas9 protein with improved genome-editing specificity. Nat. Chem. Biol. 11, 316-318 (2015).

One conceptual implementation of MERFISH is to label every target RNA so that during each round of imaging, it either has a signal (read in binary as 1 ) or does not (read in binary as 0). Using this approach, one can uniquely distinguish each RNA species by assigning it a unique 16-bit binary code and imaging over 16 rounds of hybridization. Given that there are $2^{16}$ unique binary codes, this could allow over 65,000 unique RNAs to be imaged, which is on the scale of the human transcriptome. However, Zhuang notes that in practice "the idea is a bit too rosy to be true": the accumulated error over 16 rounds of imaging makes unique RNAs nearly impossible to identify. To overcome this limitation, the team modified an error-robust encoding idea from information theory, in which any valid binary code must differ from another code by more than one bit, making it impossible for codes with a single error to be misinterpreted as alternate RNA species.

To demonstrate MERFISH, the team probed 140 genes using a 16-bit code with a Hamming distance of 4, meaning that every code differed from another by at least four bits. This method allowed the team to both identify and correct errors, yielding very accurate measurements when compared to both single-cell RNA sequencing studies and conventional smFISH. They also imaged 1,000 RNAs in single cells using a 14-bit code with a Hamming distance of 2. In this case, errors could be identified but not corrected.

Zhuang and her team are working on increasing the multiplexing capability of the method and increasing the number of cells that can be analyzed. In addition, they have begun using MERFISH to ask biological questions such as what changes occur in the transcriptome during embryo development. Zhuang is also collaborating with Catherine Dulac at Harvard University to use MERFISH to help elucidate different cell types in the hypothalamus and study their transcriptomes under different conditions. Rita Strack 